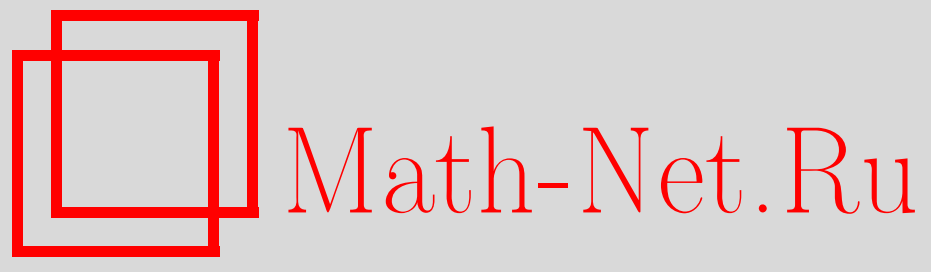

А. С. Романов, Дипольное приближение в динамике трех вихрей, ТМФ, 2007, том 150, номер 3, 409-416

DOI: https://doi.org/10.4213/tmf5987

Использование Общероссийского математического портала Math-Net.Ru подразумевает, что вы прочитали и согласны с пользовательским соглашением http://www . mathnet.ru/rus/agreement

Параметры загрузки:

IP: 54.172 .240 .79

26 апреля 2023 г., 12:47:55

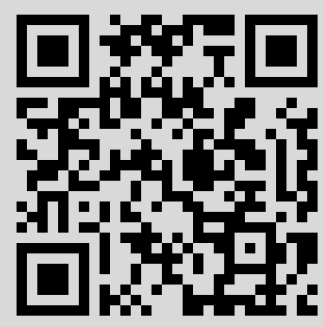




\section{ДИПОЛЬНОЕ ПРИБЛИЖЕНИЕ В ДИНАМИКЕ ТРЕХ ВИХРЕЙ}

Решена задача о взаимном движении двух близких вихрей (дипольной пары) и третьего вихря для различных функций тока.

Ключевые слова: динамика трех вихрей, дипольный вихрь, интегрируемые системы.

\section{1. ПОСТАНОВКА ЗАДАЧИ}

В настоящей работе исследуется система трех точечных вихрей, при этом предполагается, что пара вихрей обладает равными, но противоположными по знаку зарядами $-q_{1}=q_{2}=1$, т.е. образует дипольный вихрь (см. рис. 1). Третий вихрь

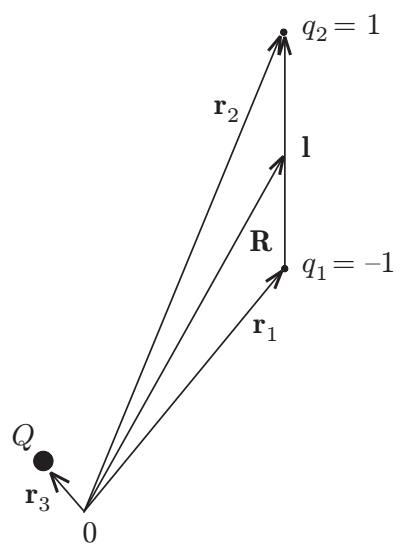

Рис. 1. Дипольный вихрь в поле вихря $Q$.

обладает зарядом $q_{3}=Q>0$ (смена знака заряда $Q$ на противоположный равносильна перемене знака диполя $\mathbf{l}=\mathbf{r}_{2}-\mathbf{r}_{1}$ на $\left.-\mathbf{l}\right)$. Движение точечных вихрей описывается гамильтоновой системой (см., например, [1], [2]) с гамильтонианом

$$
H_{0}=-Q \psi\left(\mathbf{r}_{1}-\mathbf{r}_{3}\right)+Q \psi\left(\mathbf{r}_{2}-\mathbf{r}_{3}\right)-\psi\left(\mathbf{r}_{2}-\mathbf{r}_{1}\right),
$$

где $\psi$ - функция тока. Движение вихрей такого типа возникает в разнообразных

*Российский научный центр "Курчатовский институт", Москва, Россия. E-mail: romanov@dap.kiae.ru 
задачах гидродинамики [2], физики сверхпроводников [3], физики плазмы [4]. Функция тока задается с помощью, например, логарифмических или степенных функций. Динамика вихрей определяется системой гамильтоновых уравнений

$$
\begin{aligned}
q_{k} \frac{d x_{k}}{d t} & =\frac{\partial H_{0}}{\partial y_{k}}, \\
q_{k} \frac{d y_{k}}{d t} & =-\frac{\partial H_{0}}{\partial x_{k}},
\end{aligned}
$$

Эта система обладает тремя дополнительными интегралами: это момент

$$
M=Q \mathbf{r}_{3}^{2}-\mathbf{r}_{1}^{2}+\mathbf{r}_{2}^{2}
$$

и две компоненты импульса (для удобства импульс повернут на $90^{\circ}$ в плоскости $(x, y)$ относительно оси $z)$

$$
\mathbf{P}=Q \mathbf{r}_{3}-\mathbf{r}_{1}+\mathbf{r}_{2}
$$

Как видно из выражения (1), часть гамильтониана, отвечающая за взаимодействие вихрей в дипольной паре, зависит только от $l$. Для дальнейших рассмотрений удобно ввести новую переменную $\mathbf{R}=\left(\mathbf{r}_{1}+\mathbf{r}_{2}\right) / 2$ - центр масс диполя (см. рис. 1$)$.

Заметим, что интегралы (3) и (4) не инволютивны, но из них можно составить два интеграла в инволюции [1]. Тогда из теоремы Лиувилля следует, что система трех вихрей интегрируема в квадратурах [5]. Тем не менее явной формулы для системы (2) в общем случае еще не получено (см., например, [1], [6], [7]).

В данной работе исследуется частично линеаризованный гамильтониан, который соответствует задаче о взаимном движении дипольного и монопольного вихрей. Задача заключается в явном описании взаимной динамики вихревой пары и вихря $Q$, а также в ответе на вопрос, когда данная вихревая пара устойчива к распаду на отдельные вихри. Под распадом вихревой пары подразумевается, что вихри расходятся на расстояние $l$, сравнимое с расстоянием до центрального вихря $R$.

\section{2. РАЗЛОЖКНИЕ ПО МАЛОМУ ПАРАМЕТРУ $l / R$}

Произведем частичную линеаризацию гамильтониана (1) по малому параметpy $l / R$. Редукция приводит к гамильтоновой системе, которая описывает динамику диполя при условии, что параметр диполя $l$ остается малым по сравнению с $R$ в течение всего времени динамики системы.

Пусть в начальный момент времени вихрь с зарядом $Q$ находится в начале системы координат $\mathbf{r}_{3}(0)=0$. Выразим из соотношения (4) $\mathbf{r}_{3}$ и подставим в интеграл для момента (3), записанного в новых переменных $\mathbf{R}, \mathbf{l}$ :

$$
M=2(\mathbf{R}, \mathbf{l})+O\left(l^{2}\right) \approx 2(\mathbf{R}, \mathbf{l}) .
$$

Гамильтониан (1) в новых переменных имеет вид

$$
H_{0}=-Q \psi\left(\mathbf{R}-\frac{\mathbf{l}}{2}-\frac{\mathbf{l}_{0}-\mathbf{l}}{Q}\right)+Q \psi\left(\mathbf{R}+\frac{\mathbf{l}}{2}-\frac{\mathbf{l}_{0}-\mathbf{l}}{Q}\right)-\psi(\mathbf{l}) .
$$

После линеаризации первых двух слагаемых в (6) гамильтониан (1) записывается в виде

$$
H=Q(\mathbf{R}, \mathbf{l}) \frac{\psi^{\prime}(R)}{R}-\psi(l),
$$


где слагаемое $O\left(l^{2}\right)$ отбрасывается. В выражении для гамильтониана (7) не учтена динамика центрального вихря $Q$. Это связано с тем, что при редукции точного гамильтониана (6) вклад, связанный с учетом динамики вихря $Q$, также квадратичен по $l$. Тем не менее динамику центрального вихря $Q$ можно определить из закона сохранения импульса (4):

$$
\mathbf{r}_{3}=\frac{\mathbf{l}_{0}-\mathbf{l}}{Q}
$$

Гамильтониан (7) включает в себя интеграл момента (5), поэтому удобно обозначить $(\mathbf{R}, \mathbf{l})=M$. В результате для переменных $\mathbf{R}, \mathbf{l}$ получается гамильтонова система в стандартной форме:

$$
\begin{aligned}
\dot{R}_{x} & =\frac{\partial H}{\partial l_{y}}=Q \frac{\psi^{\prime}(R)}{R} R_{y}-\frac{\psi^{\prime}(l)}{l} l_{y}, \\
\dot{R}_{y} & =-\frac{\partial H}{\partial l_{x}}=-Q \frac{\psi^{\prime}(R)}{R} R_{x}+\frac{\psi^{\prime}(l)}{l} l_{x}, \\
\dot{l}_{x} & =\frac{\partial H}{\partial R_{y}}=Q M\left(\psi^{\prime \prime}(R)-\frac{\psi^{\prime}(R)}{R}\right) \frac{R_{y}}{R^{2}}+Q \frac{\psi^{\prime}(R)}{R} l_{y}, \\
\dot{l}_{y} & =-\frac{\partial H}{\partial R_{x}}=-Q M\left(\psi^{\prime \prime}(R)-\frac{\psi^{\prime}(R)}{R}\right) \frac{R_{x}}{R^{2}}-Q \frac{\psi^{\prime}(R)}{R} l_{x},
\end{aligned}
$$

где гамильтониан $H$ задан выражением (7). Система уравнений (9) описывает динамику центра масс диполя $\mathbf{R}$ и изменение дипольного момента $\mathbf{l}$. Домножим первое уравнение на $R_{x}$, а второе - на $R_{y}$, сложим их. Аналогичные операции проведем с третьим и четвертым уравнениями в $(9)$. В результате получим систему уравнений

$$
\begin{aligned}
\frac{d R}{d t} & =-\frac{\psi^{\prime}(l)}{l R} T, \\
\frac{d l}{d t} & =-\frac{Q M}{l R^{2}}\left(\psi^{\prime \prime}(R)-\frac{\psi^{\prime}(R)}{R}\right) T, \\
\frac{d T}{d t} & =-Q M\left(\psi^{\prime \prime}(R)-\frac{\psi^{\prime}(R)}{R}\right)-\psi^{\prime}(l), \\
T & =R_{x} l_{y}-R_{y} l_{x},
\end{aligned}
$$

где в уравнениях для $\dot{R}, \dot{l}$ естественным образом появилась новая переменная $T=$ $R_{x} l_{y}-R_{y} l_{x}$. Вместо четырех уравнений (9) получается три уравнения на переменные $R, l, T$. Гамильтониан (7) связывает переменные $R$ и $l$, поэтому, выразив $R$ через $l$, можно сократить количество переменных до двух и в итоге проинтегрировать систему. В общем виде выразить $R$ из (7) через $l$ не удастся, но для ряда конкретных функций тока $\psi$ это сделать можно.

2.1. Убывающая степенная функция тока. Выберем функцию тока в виде $\psi(r)=1 / r^{n}$. Гамильтониан (7) для такой функции тока есть

$$
H=-\frac{Q M n}{R^{n+2}}-\frac{1}{l^{n}}, \quad M=(\mathbf{R}, \mathbf{l}) .
$$


Получаем следующее выражение $R(l)$ для гамильтониана (11):

$$
\begin{gathered}
R(l)=\left(-\frac{Q M n}{H+1 / l^{n}}\right)^{1 /(n+2)}, \\
\text { 1) } M<0 \rightarrow\left\{\begin{array}{lll}
H>0 \quad \Rightarrow \quad 0 \leqslant l<\infty, \\
H<0 \quad \Rightarrow & 0 \leqslant l<1 /(-H)^{1 / n},
\end{array}\right. \\
\text { 2) } M>0 \rightarrow H<0 \Rightarrow l>1 /(-H)^{1 / n},
\end{gathered}
$$

где для интегралов $M$ и $H$ выписаны условия, при которых возможно получить $R(l)$. Уравнения (10) после подстановки (12) сводятся к двум уравнениям на переменные $l$ и $T$ :

$$
\begin{aligned}
\frac{d l}{d t} & =-\frac{Q M n(n+2)}{l}\left(-\frac{H+1 / l^{n}}{Q M n}\right)^{(n+4) /(n+2)} T, \\
\frac{d T}{d t} & =H(n+2)+\frac{2(n+1)}{l^{n}} .
\end{aligned}
$$

Точка равновесия $(i=\dot{T}=0)$ системы (13) определяется как

$$
R^{*}=\left(-\frac{2 Q M(n+1)}{H}\right)^{1 /(n+2)}, \quad l^{*}=\left(-\frac{2(n+1)}{(n+2) H}\right)^{1 / n}, \quad T^{*}=0 .
$$

Здесь использовано условие (12) для нахождения особой точки $R^{*}$. Особая точка для системы (10) является седловой и существует при условии $M>0, H<0$. Проинтегрировав систему уравнений (13), получим

$$
T^{2}-T_{0}^{2}=l^{2}\left(-\frac{Q M n}{H+1 / l^{n}}\right)^{2 /(n+2)}-l_{0}^{2}\left(-\frac{Q M n}{H+1 / l_{0}^{n}}\right)^{2 /(n+2)} .
$$

На рис. 2 представлены зависимости $T(l)$ для разных начальных условий (см. (12)). В результате поставленная задача полностью решена.

Покажем, что система (13) гамильтонова. Это не вполне очевидно, поскольку динамика на гиперповерхности постоянных интегралов (в данном случае плоскости $(T, l))$ не обязана быть гамильтоновой. Чтобы показать это, сделаем замену

$$
d y=\frac{-l d l}{Q M n(n+2)\left(-\frac{H+1 / l^{n}}{Q M n}\right)^{(n+4) /(n+2)}} .
$$

Нетрудно убедиться, что система (13) в переменных $T, y$ примет стандартный вид с гамильтонианом

$$
H_{y, T}=\frac{T^{2}}{2}+G(y), \quad G(y)=-H(n+2) y-\int_{y_{0}}^{y} \frac{2(n+1)}{l(z)^{n}} d z .
$$

2.2. Логарифмическая функция тока. Такая ситуация обычно исследуется в задачах гидродинамики. Рассмотрение функции тока вида $\psi(r)=-\ln r$ делается аналогично предыдущему случаю. Гамильтониан (7) в переменных $\mathbf{R}, \mathbf{l}$ принимает вид

$$
H=-\frac{Q M}{R^{2}}+\ln l, \quad M=(\mathbf{R}, \mathbf{l}) .
$$




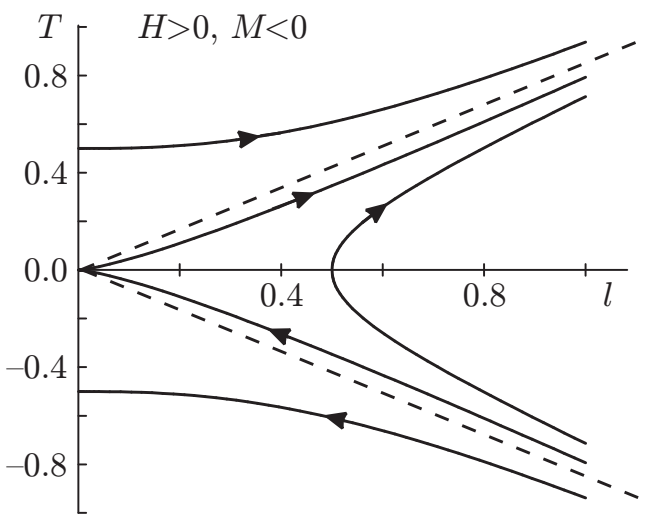

a

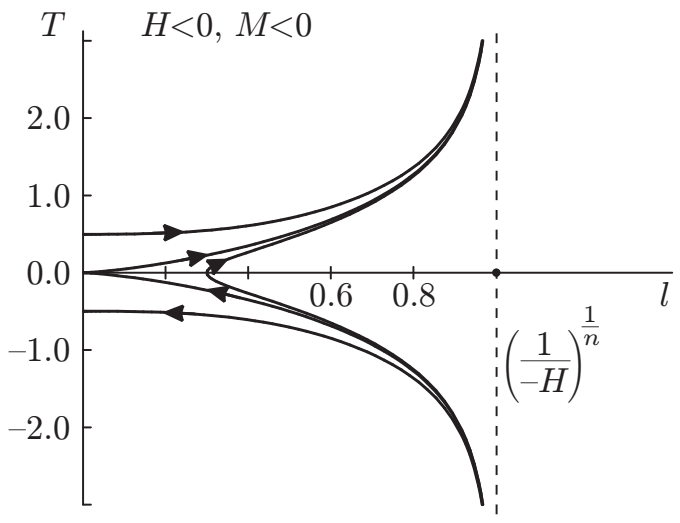

б

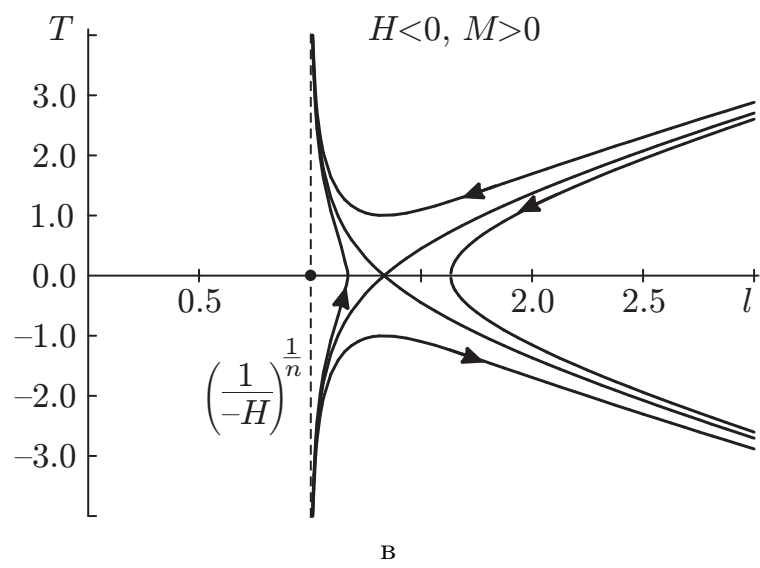

Рис. 2. Зависимости $T(l)$ для разных значений $M, H$.

Особая точка в такой системе седловая. Ситуация похожа на вариант функции тока $1 / r^{n}$. Решение в переменных $(l, T)$ есть

$$
T^{2}-T_{0}^{2}=\frac{l_{0}^{2} Q M}{H-\ln l_{0}}-\frac{l^{2} Q M}{H-\ln l} .
$$

2.3. Функция тока вида $\psi(r)=r^{n}$. Гамильтониан $(7)$ записывается в виде

$$
H=Q M n R^{n-2}-l^{n} .
$$

Для $n=2$ динамика диполя тривиальна, что следует из уравнений (9) и (10). Гамильтонова система, отвечающая функции тока $\psi(r)=r^{2}$, легко интегрируется:

$$
R(t)=\sqrt{R_{0}^{2}+4\left(l_{0} t^{2}-T_{0} t\right)}, \quad l(t)=l_{0}, \quad T(t)=-2 l_{0} t+T_{0},
$$

при этом решается как редуцированная (7), так и точная (1) модель, что связано с таким выбором функции тока. 
Случай $n=1$, возможно, интересен в связи с вопросами кварк-глюонной плазмы (КГП): в теории сильного взаимодействия потенциал взаимодействия между кварками $\varphi(r)$ пропорционален $r$ [8]. При изучении столкновений ядер вещества в ускорителях предполагается, что образуется КГП. При нецентральном столкновении может возникнуть такая ситуация, что КГП будет вращаться и возникнут сверхсильные магнитные поля. Под действием сил Лоренца и Кориолиса кварки будут двигаться с дрейфовой скоростью

$$
\mathbf{u}=A \mathbf{e}_{z} \times \nabla \varphi
$$

где $\varphi(r)$ - потенциал взаимодействия между кварками, $A-$ константа. Сравнивая скорость кварка в потенциале $\varphi(r)$ с формулой для скорости отдельного вихря в поле функции тока $\psi\left(\mathbf{u}=\mathbf{e}_{z} \times \nabla \psi\right)$, убеждаемся, что они совпадают. Функция тока $\psi(r)$ соответствует потенциалу взаимодействия $\varphi(r) \sim r$.

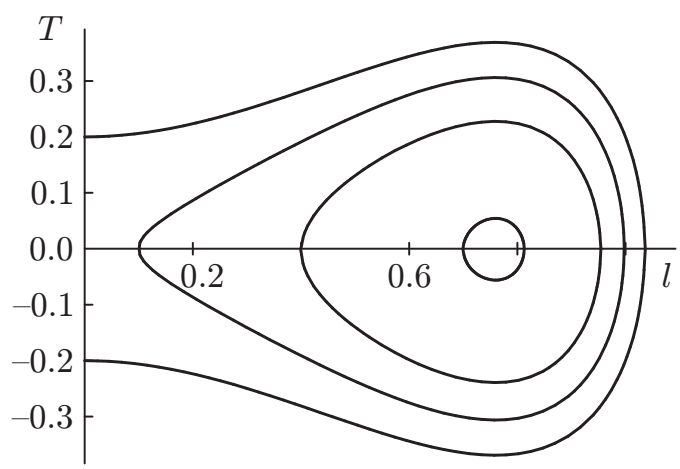

Рис. 3. Зависимость $T(l)$ для $M<0, H<0$.

Особая точка системы уравнений на переменные $R, l, T$ совпадает с определением (14) при $n$, замененном на $-n$. Из вида гамильтониана (19) можно ожидать, что для $n>2$ особая точка будет центром (например, случай $n=4, M<0$ ), а поэтому все величины будут осциллировать вблизи положения равновесия. Решение задачи о динамике диполя в поле вихря $Q$ для гамильтониана $(19)$ в переменных $(l, T)$ записывается в виде

$$
T^{2}-T_{0}^{2}=l^{2}\left(\frac{Q M n}{H+l^{n}}\right)^{2 /(2-n)}-l_{0}^{2}\left(\frac{Q M n}{H+l_{0}^{n}}\right)^{2 /(2-n)} .
$$

На рис. 3 представлена зависимость $T(l)$ для случая, когда особая точка существует.

2.4. Комбинация функций тока. Рассмотрим комбинированный вариант функций тока $1 / r^{n}$ и $r^{n}$. А именно, диполь будет характеризоваться функцией тока $\phi(l)=1 / l^{n}$, а центральный вихрь $Q-$ функцией тока $\psi(R)=R^{m}$. Гамильтониан для этих функций тока имеет вид

$$
H=Q M m R^{m-2}-\frac{1}{l^{n}} .
$$


Система уравнений, определяемых гамильтонианом (22), имеет два типа особых точек в зависимости от параметров $n, m, M, H$. Особая точка определяется как

$$
R^{*}=\left(\frac{H n}{Q M m(n-m+2)}\right)^{1 /(m-2)}, \quad l^{*}=\left(\frac{n-m+2}{(m-2) H}\right)^{1 / n}, \quad T^{*}=0 .
$$

Рассмотрим случай $M>0, m>2$. Из (23) получаем, что $(n-m+2) H>0$. Если $n-m+2>0$, особая точка является седловой, а если $n-m+2<0$, особая точка центр. Мы не будем подробно останавливаться на классификации поведения такой системы, поведение во многом схоже с ранее рассмотренными случаями. Отметим, что система (22) включает в себя как частные случаи функции тока $1 / r^{n}, r^{n}$.

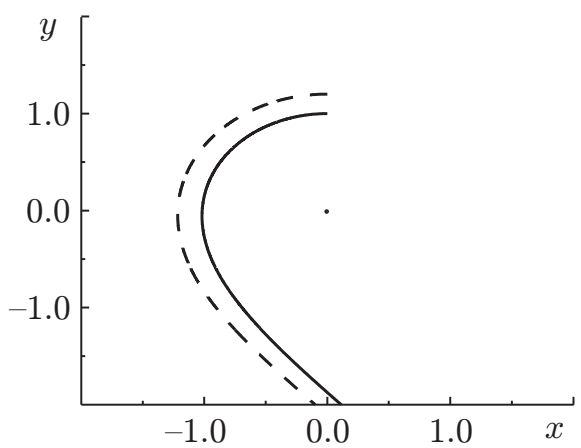

a

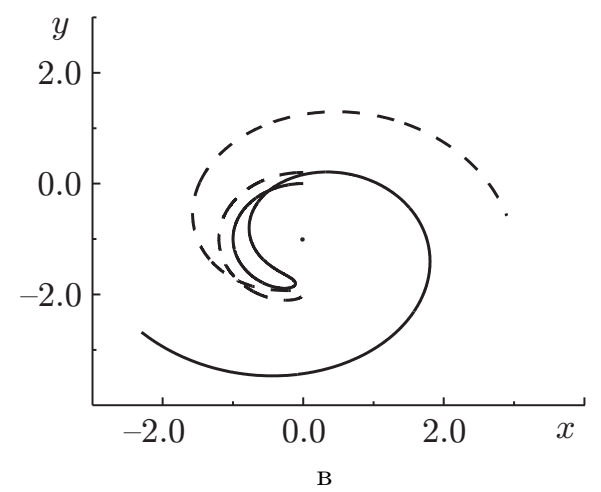

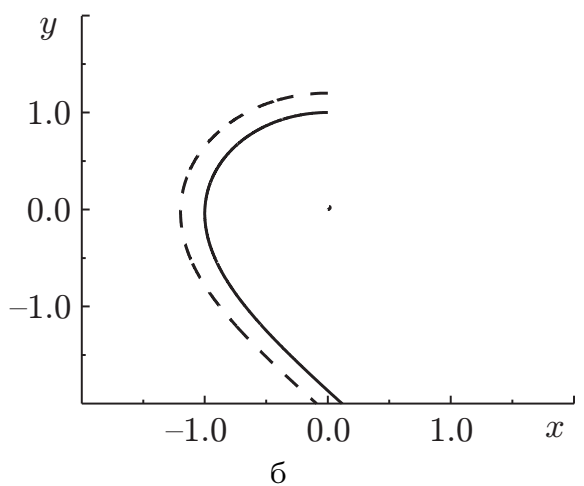

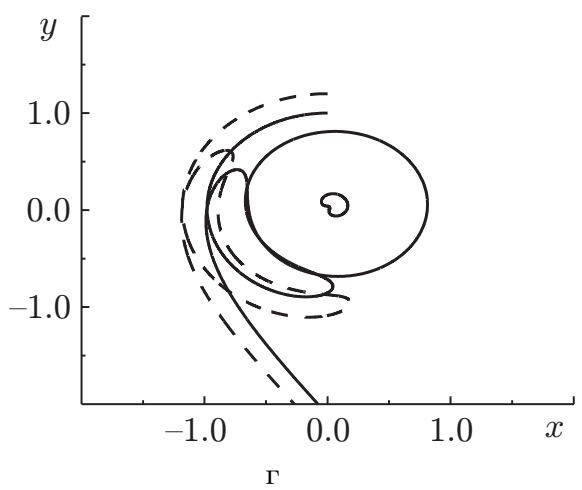

Рис. 4. Динамика диполя в поле вихря для функции тока $\psi(r)=1 / r$. В центре расположен вихрь с $Q=10$. Устойчивость диполя для редуцированного и точного гамильтониана $(\mathrm{a}$, б); распад диполя для редуцированного гамильтониана (в); распад и повторное образование диполя для точного гамильтониана (г).

2.5. Сравнение с точным гамильтонианом. При численных расчетах было выяснено, что точная (1) и редуцированная (7) системы ведут себя похожим образом в области параметров, где диполь устойчив к распаду (см. рис. 4а, б). Теперь перейдем через особую точку на рис. 2в в область неустойчивого поведения диполя, увеличив начальное расстояние $l$ между вихрями в диполе. В редуцированной 
модели (см. рис. 4в) происходит распад вихревой пары на пару вихрей, которые по спирали раскручиваются. Для случая точной динамики картина иная (см. рис. 4г). Сначала вихревая пара распадается $(l \approx R)$, вихрь, ближайший к центральному вихрю $Q$, делает оборот вокруг него, после чего опять образуется вихревая пара, которая уходит на бесконечность (возможен вариант колебаний, когда дипольный вихрь периодически то распадается, то образуется вблизи центрального вихря).

\section{3. ЗАКЛЮЧЕНИЕ}

В работе рассмотрена взаимная динамика дипольного вихря в поле центрального вихря. Задача состояла в том, чтобы описать эволюцию диполя и указать область начальных параметров системы, для которых размер диполя не меняется существенно по сравнению с расстоянием до центрального вихря $Q$. Для решения поставленной задачи точный гамильтониан (1) с учетом всех четырех интегралов частично линеаризовался по параметру диполя $l / R$. После редукции гамильтониан принял вид (7) для переменных $(\mathbf{R}, \mathbf{l})$. Редуцированная система точно проинтегрирована для разных функций тока. Численные расчеты показали, что редуцированная модель предсказывает время первого распада дипольного вихря и приемлемо описывает динамику дипольной пары в случае отсутствия распада.

Благодарности. Автор благодарен К.В. Чукбару за поставленную задачу и интересные беседы, стимулировавшие решение задачи. Также автор благодарен С. Ю. Доброхотову за ценные замечания по работе, которые помогли улучшить изложение материала. Работа выполнена при поддержке Программы поддержки ведущих научных школ (грант № НШ-2292.2003.2) и системы инициативных проектов РНЦ "Курчатовский институт".

\section{Список литературы}

[1] А. В. Борисов, И. С. Мамаев, М. А. Соколовский (ред.), Фундаменталъные и прикладные проблемь теории вихрей, Институт компьютерных исследований, М.-Ижевск, 2003.

[2] Ф. Дж. Сэффмэн, Динамика вихрей, Научный мир, М., 2000.

[3] G. Blatter, M. V. Feigel'man, V.B. Geshkenbein, et al., Rev. Mod. Phys., 66 (1994), 1125.

[4] А. С. Кингсеп, К. В. Чукбар, В.В.Яньков, "Электронная магнитная гидродинамика", Вопросы теории плазмы, № 16, ред. Б. Б. Кадомцев, Энергоатомиздат, М., 1987, 209.

[5] В.И. Арнольд, Математические методы классической механики, Эдиториал УРСС, M., 2003.

[6] Е. А. Новиков, ЖЭЭФФ, 68 (1975), 1868.

[7] H. Aref, Phys. Fluids, 22 (1979), 393.

[8] Л. Б. Окунь, Физика элементарных частии, Наука, М., 1988.

Поступила в редакцию 17.04.2006, после доработки 3.07.2006 